\title{
Synthesis of $N$-[2-(1-Piperidinyl)ethyl]benzamides
}

\author{
Fumiko FusisaKi, ${ }^{*}$ Nobuhiro Abe, and Kunihiro Sumoto \\ Faculty of Pharmaceutical Science, Fukuoka University, Nanakuma, Jonann-ku, Fukuoka 814-0180, Japan. \\ Received July 30, 2001; accepted October 12, 2001
}

Novel benzamide derivatives, $N$-[1-(aminocarbonyl)-2-(1-piperidinyl)ethyl]benzamides (4 and 5), were prepared from the reaction of $\beta$-piperidinoalanine (6) as the starting material. \author{
reaction \\ Key words $\beta$-piperidinoalanine; benzamide derivative; Mannich base; mixed anhydride; pivaloyl chloride; Schotten-Baumann
}

In the course of our studies on the chemistry of enaminoketones, ${ }^{1)}$ we synthesized some enamino-ketone Mannich base derivatives depicted by the general formula 1 (Chart 1), and found that most had strong opioid analgesic activity.2) Among the derivatives synthesized, compound 1a or $\mathbf{1 b}$ showed higher analgesic acitivity than that of morphine, and both compounds had various pharmacological activities such as sedative, antitussive, or anticonvulsant activities. These results suggested that $\mathbf{1 a}$ or $\mathbf{1 b}$ can interact with a variety of receptors in the central nervous system. On the basis of those studies, we attempted the chemical modification of the prototype compound 1 as shown in Chart 2. Thus the alicyclic moiety (part A enclosed in the dotted lines in 1) was removed and the vinylogous amide moiety was divided into two amide moieties (parts B and C in structure 2), leading to the malonodiamide Mannich base 2. However, the synthesized compounds 2 were unstable. Therefore we planned to synthesize the Mannich base $\mathbf{3}$ in which the anilide moiety (part $\mathrm{C}$ in 2) was replaced with a benzamide group. This molecule 3 bears a marked structural resemblance to some dopamine antagonists. In this paper, we report the synthesis of novel benzamide derivatives ( $\mathbf{4}$ and $\mathbf{5}$ in Chart 3 ) with the general structure 3 .

\section{Results and Discussion}

For the preparation of the target compounds $\mathbf{4}$ and $\mathbf{5}$ starting from $\beta$-piperidinoalanine $\mathbf{6}$, two rational synthetic pathways via amidation of this amino acid are possible. The efficient synthesis of starting material $\mathbf{6}$ and related compounds has already been reported. ${ }^{3)}$ To begin, we attempted the procedure via amidation of the carboxylic acid functionality. Thus treatment of $\mathbf{6}$ with methanolic hydrogen chloride gave the corresponding amino acid methyl ester 7 in excellent yield. The ester dihydrochloride 7 was reacted with methylamine and pyrrolidine to give corresponding amides 8 and 9 ( 87 and $72 \%$ yield, respectively), which were used in next

step without further purification. Treatment of compound $\mathbf{8}$ with carboxylic acids in the presence of dicyclohexylcarbodiimide (DCC) gave the desired benzamide derivative 4. In a similar manner, the reaction of $\mathbf{9}$ with carboxylic acids afforded corresponding benzamide derivatives $\mathbf{5 a}-\mathbf{d}$. Since no reaction of 7 with piperidine occurred, ${ }^{4)}$ alternative amidation of the target compound 5e was investigated and we found that a method via mixed anhydride was efficient. Thus the reaction of $\mathbf{6}$ with benzoyl chloride under Schotten-Baumann reaction conditions gave $N$-[(1-carboxy)-2-(1-piperidinyl)ethyl]benzamide $\mathbf{1 0}$ in good yield. The reactant of $\mathbf{1 0}$ with pivaloyl chloride, giving a corresponding mixed anhydride, was allowed to react with piperidine to provide the desired $\mathbf{5 e}$ in $50 \%$ yield. Using this procedure, compound $\mathbf{5 a}$ was also obtained from $\mathbf{1 0}$ and pyrrolidine in 63\% yield (see Chart 3). This method via mixed anhydride was also applicable for the preparation of $\mathbf{4}$ and $\mathbf{5 b}-\mathbf{d}$ and provides a new synthetic procedure for this class of compounds. The results are summarized in Table 1.5)

The molecules of $\mathbf{4}$ and $\mathbf{5}$ synthesized above have frameworks similar to those of tiapride ${ }^{6}$ and metoclopramide ${ }^{7)}$
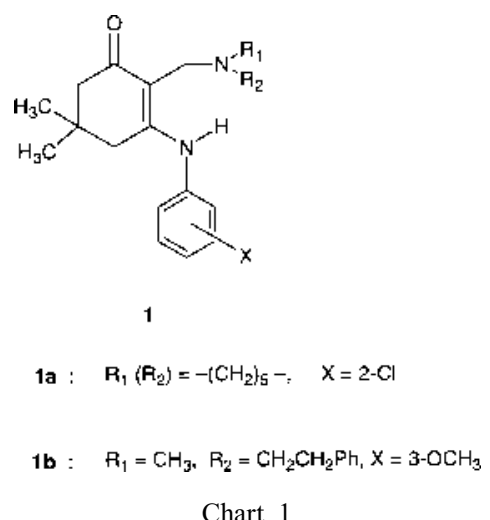

Chart 1

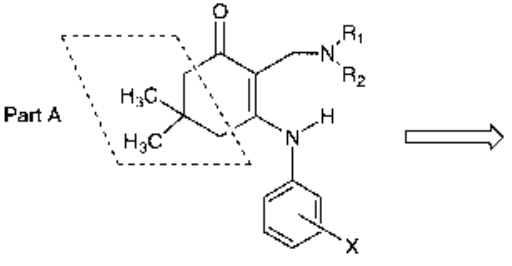

1

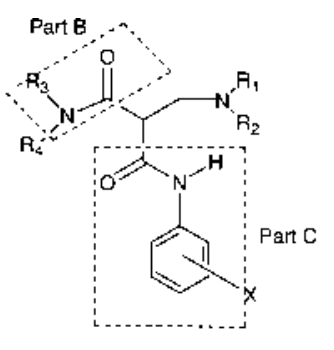

$\mathbf{z}$

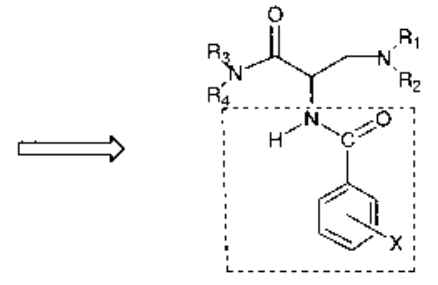

ง

Chart 2 


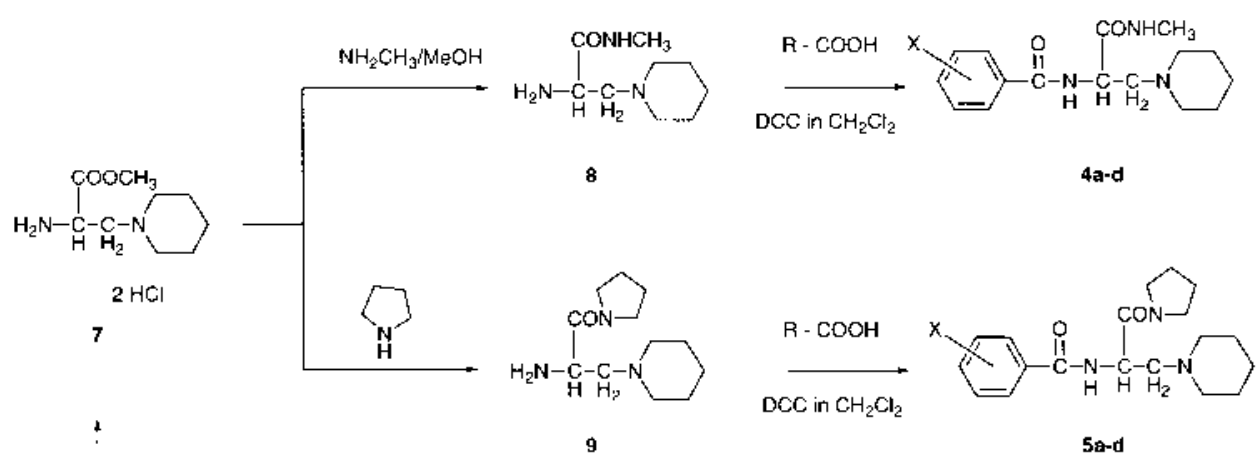

$\mathrm{H}^{+} /$MeOH
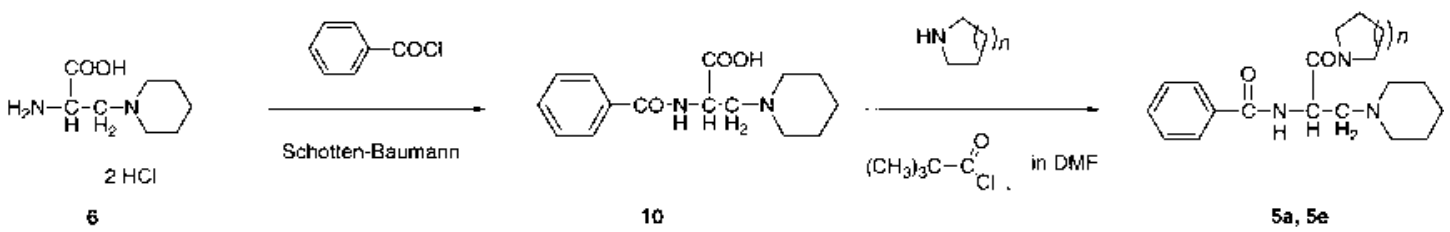

10

5a, 5e

Chart 3

Table 1. Preparation of $N$-[(1-Aminocarbonyl)-2-(1-piperidinyl)ethyl]benzamides $\mathbf{4}$ and $\mathbf{5}$

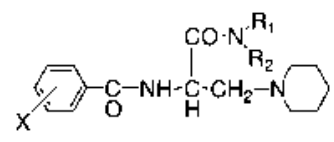

\begin{tabular}{|c|c|c|c|c|c|c|c|c|}
\hline \multirow{2}{*}{$\begin{array}{l}\text { Compd. } \\
\text { no. }\end{array}$} & \multirow{2}{*}{$X$} & \multirow{2}{*}{$-N^{\mathrm{R}_{1}}$} & \multirow{2}{*}{$\begin{array}{l}\mathrm{mp}\left({ }^{\circ} \mathrm{C}\right) \\
\text { (Solvent) }\end{array}$} & \multirow{2}{*}{ Formula } & \multirow{2}{*}{$\begin{array}{l}\text { FAB-MS } \\
\left(\mathrm{MH}^{+}\right)\end{array}$} & \multicolumn{3}{|c|}{ Analysis (\%) Calcd (Found) } \\
\hline & & & & & & $\mathrm{C}$ & $\mathrm{H}$ & $\mathrm{N}$ \\
\hline $4 a$ & $\mathrm{H}$ & $-\mathrm{NHCH}_{3}$ & $\begin{array}{l}139-142 \\
\text { (AcOEt) }\end{array}$ & $\mathrm{C}_{16} \mathrm{H}_{23} \mathrm{~N}_{3} \mathrm{O}_{2}$ & 290 & $\begin{array}{r}66.41 \\
(66.51\end{array}$ & $\begin{array}{l}8.01 \\
8.03\end{array}$ & $\begin{array}{l}14.52 \\
14.54)\end{array}$ \\
\hline $4 b$ & $\begin{array}{l}2-\mathrm{OCH}_{3}, 4-\mathrm{NH}_{2}, \\
5-\mathrm{Cl}\end{array}$ & $-\mathrm{NHCH}_{3}$ & $\begin{array}{l}183 \text { (dec.) } \\
\left(\mathrm{H}_{2} \mathrm{O}\right)\end{array}$ & $\begin{array}{l}\mathrm{C}_{17} \mathrm{H}_{25} \mathrm{ClN}_{4} \mathrm{O}_{3} \\
\cdot \mathrm{HCl} \cdot \mathrm{H}_{2} \mathrm{O}\end{array}$ & 369 & $\begin{array}{r}48.23 \\
(48.10\end{array}$ & $\begin{array}{l}6.67 \\
6.61\end{array}$ & $\begin{array}{l}13.23 \\
13.20)\end{array}$ \\
\hline $4 c$ & $3,4-\mathrm{OCH}_{3}$ & $-\mathrm{NHCH}_{3}$ & $\begin{array}{l}154-155 \\
\left(\mathrm{CH}_{3} \mathrm{CN}\right)\end{array}$ & $\mathrm{C}_{18} \mathrm{H}_{27} \mathrm{~N}_{3} \mathrm{O}_{4}$ & 350 & $\begin{array}{r}61.87 \\
(61.95\end{array}$ & $\begin{array}{l}7.79 \\
7.71\end{array}$ & $\begin{array}{l}12.03 \\
12.04)\end{array}$ \\
\hline $4 d$ & $3,4,5-\mathrm{OCH}_{3}$ & $-\mathrm{NHCH}_{3}$ & $\begin{array}{l}138-141 \\
(\mathrm{AcOEt})\end{array}$ & $\mathrm{C}_{19} \mathrm{H}_{29} \mathrm{~N}_{3} \mathrm{O}_{5}$ & 380 & $\begin{array}{r}60.14 \\
(59.96\end{array}$ & $\begin{array}{l}7.7 \\
7.90\end{array}$ & $\begin{array}{l}11.07 \\
11.04)\end{array}$ \\
\hline $5 \mathbf{a}$ & $\mathrm{H}$ & & $\begin{array}{l}216-218(\mathrm{dec} .) \\
\left(\mathrm{CH}_{3} \mathrm{CN}\right)\end{array}$ & $\begin{array}{l}\mathrm{C}_{19} \mathrm{H}_{27} \mathrm{~N}_{3} \mathrm{O}_{2} \\
\cdot \mathrm{HCl}\end{array}$ & 330 & $\begin{array}{r}62.37 \\
(62.14\end{array}$ & $\begin{array}{l}7.71 \\
7.76\end{array}$ & $\begin{array}{l}11.48 \\
11.63)\end{array}$ \\
\hline $5 b$ & $\begin{array}{l}2-\mathrm{OCH}_{3}, 4-\mathrm{NH}_{2}, \\
5-\mathrm{Cl}\end{array}$ & & $\begin{array}{l}175-177 \\
\text { (Acetone) }\end{array}$ & $\mathrm{C}_{20} \mathrm{H}_{29} \mathrm{ClN}_{4} \mathrm{O}_{3}$ & 409 & $\begin{array}{r}58.74 \\
(58.76\end{array}$ & $\begin{array}{l}7.15 \\
7.18\end{array}$ & $\begin{array}{l}13.7 \\
13.63)\end{array}$ \\
\hline $5 c$ & $3,4-\mathrm{OCH}_{3}$ & & $\begin{array}{l}106-112 \\
(\mathrm{AcOEt})\end{array}$ & $\mathrm{C}_{21} \mathrm{H}_{31} \mathrm{~N}_{3} \mathrm{O}_{4}$ & 390 & $\begin{array}{r}64.76 \\
(64.46\end{array}$ & $\begin{array}{l}8.02 \\
8.03\end{array}$ & $\begin{array}{l}10.79 \\
10.52)\end{array}$ \\
\hline 5d & $3,4,5-\mathrm{OCH}_{3}$ & & $\begin{array}{l}159-162 \\
\left(\mathrm{CH}_{3} \mathrm{CN}\right)\end{array}$ & $\mathrm{C}_{22} \mathrm{H}_{33} \mathrm{~N}_{3} \mathrm{O}_{5}$ & 420 & $\begin{array}{r}62.99 \\
(62.90\end{array}$ & $\begin{array}{l}7.93 \\
7.84\end{array}$ & $\begin{array}{l}10.02 \\
10.15)\end{array}$ \\
\hline $5 e^{a)}$ & $\mathrm{H}$ & $1-$ & $\begin{array}{l}161-162 \\
\left(\mathrm{CH}_{3} \mathrm{CN}\right)\end{array}$ & $\mathrm{C}_{20} \mathrm{H}_{29} \mathrm{~N}_{3} \mathrm{O}_{2}$ & 344 & $\begin{array}{r}69.94 \\
(69.86\end{array}$ & $\begin{array}{l}8.51 \\
8.50\end{array}$ & $\begin{array}{l}12.23 \\
12.21)\end{array}$ \\
\hline
\end{tabular}

a) This compound was obtained by the method via mixed anhydride, other compounds were obtained by the method via ester 7 .

(Chart 4), which are clinically useful dopamine receptor antagonists. Molecular model examination of these compounds ${ }^{8)}$ suggested the possibility that compounds $\mathbf{4}$ and $\mathbf{5}$ may interact with the dopamine receptor. Further synthetic applications and pharmacological evaluations of the compounds synthesized are under investigation to find a new candidate with potent dopamine antagonist activity.

\section{Experimental}

Melting points were uncorrected. Infrared (IR) spectra were measured with a Shimadzu FTIR-8100 spectrometer. ${ }^{1} \mathrm{H}-(500 \mathrm{MHz})$ and ${ }^{13} \mathrm{C}-(125$ $\mathrm{MHz}$ ) NMR spectra were obtained with a JEOL JNM A-500. Chemical

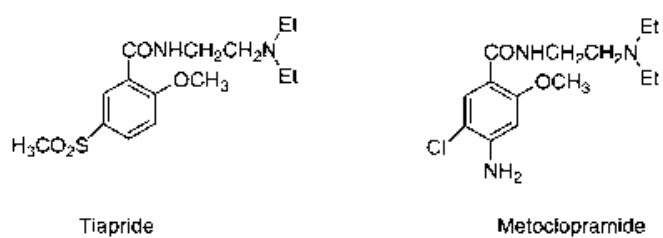

Chart 4

shifts were expressed as $\delta$ ppm downfield from an internal tetramethylsilane (TMS) signal $(0 \mathrm{ppm})$ or sodium 3-(trimethylsilyl)propionic 2,2,3,3- $d_{4}$ acid (TSP) signal $(0 \mathrm{ppm})\left(\right.$ in $\left.\mathrm{D}_{2} \mathrm{O}\right)$ for ${ }^{1} \mathrm{H}-\mathrm{NMR}$, and the carbon signal of the 
corresponding solvent [CDCl $(77.0 \mathrm{ppm})$, DMSO- $\left.d_{6}(39.5 \mathrm{ppm})\right]$, and TSP $(0 \mathrm{ppm})$ (in $\mathrm{D}_{2} \mathrm{O}$ ) for ${ }^{13} \mathrm{C}-\mathrm{NMR}$, respectively. The signal assignments were confirmed with ${ }^{1} \mathrm{H}-{ }^{1} \mathrm{H}$ correlation spectroscopy (COSY), ${ }^{1} \mathrm{H}-{ }^{13} \mathrm{C}$ heteronuclear multiple quantum coherence (HMQC), and ${ }^{1} \mathrm{H}-{ }^{13} \mathrm{C}$ heteronuclear multiple bond connectivity (HMBC) spectra. FAB-MS were obtained with a JEOL JMS-HX110 mass spectrometer. Yield and physical data (MS, analysis) for compounds $\mathbf{4}$ and $\mathbf{5}$ are summarized in Table 1. Other spectroscopic data are recorded below.

Methyl $\alpha$-Amino-1-piperidinepropanoate Dihydrochloride (7) Methanolic $\mathrm{HCl}(10 \%)$ was added to a solution of 6 in anhydrous methanol and the mixture was kept at room temperature for $24 \mathrm{~h}$, then evaporated to dryness in vacuo. After a few such treatments with methanolic $\mathrm{HCl}$, the residue finally crystallized completely. The yield was quantitative. Recrystallization from methanol gave an analytical sample of 7. This compound was colorless prisms and quite hygroscopic, $\mathrm{mp} 178^{\circ} \mathrm{C}(\mathrm{dec}$.). IR ( $\mathrm{KBr})$ : $1759 \mathrm{~cm}^{-1}$. Negative FAB-MS m/z: $257(\mathrm{M}+2 \mathrm{HCl}-\mathrm{H})^{-} .{ }^{1} \mathrm{H}-\mathrm{NMR}$ (DMSO$\left.d_{6}\right) \delta: 1.55\left(2 \mathrm{H}, \mathrm{brs}, 4-\mathrm{CH}_{2}\right.$ of piperidine ring $), 1.81-1.86\left(4 \mathrm{H}, \mathrm{m}, 3,5-\mathrm{CH}_{2}\right.$ of piperidine ring), $3.17-3.51\left[3 \mathrm{H}, \mathrm{m}, 2-\mathrm{CH}_{2}\right.$ of piperidine ring and $\left.\mathrm{NH}_{2} \mathrm{CH}(\mathrm{CON}=) \mathrm{CH} H \mathrm{HN}=\right], 3.64-3.70\left[3 \mathrm{H}, \mathrm{m}, 6-\mathrm{CH}_{2}\right.$ of piperidine ring and $\left.\mathrm{NH}_{2} \mathrm{CH}(\mathrm{CON}=) \mathrm{CHHN}=\right], 3.79\left(3 \mathrm{H}, \mathrm{s}, \mathrm{CH}_{3}\right), 4.90[1 \mathrm{H}, \mathrm{q}, J=4 \mathrm{~Hz}$, $\left.\mathrm{NH}_{2} \mathrm{C} \underline{\mathrm{H}}(\mathrm{CON}=) \mathrm{CH}_{2} \mathrm{~N}=\right], 9.64\left(2 \mathrm{H}, \mathrm{br}, \mathrm{NH}_{2}\right) .{ }^{13} \mathrm{C}-\mathrm{NMR}\left(\mathrm{DMSO}-d_{6}\right) \delta$ : 20.9 (C4 of piperidine ring), 22.3 ( $\mathrm{C} 3, \mathrm{C} 5$ of piperidine ring), 47.8 $\left[\mathrm{NH}_{2} \mathrm{C} H(\mathrm{CON}=) \mathrm{CH}_{2}-\right], 53.2$ (C2 of piperidine ring), $53.4\left(\mathrm{CH}_{3}\right), 55.6$ $\left(\mathrm{NH}_{2} \mathrm{CH}(\mathrm{CON}=) \mathrm{CH}_{2}-\right), 56.0$ (C6 of piperidine ring), $166.6(\mathrm{CO})$. Anal. Calcd for $\mathrm{C}_{9} \mathrm{H}_{18} \mathrm{~N}_{2} \mathrm{O}_{2} \cdot 2 \mathrm{HCl} \cdot 0.1 \mathrm{H}_{2} \mathrm{O}: \mathrm{C}, 41.42 ; \mathrm{H}, 7.80 ; \mathrm{N}, 10.73$. Found: $\mathrm{C}$, $41.20 ; \mathrm{H}, 7.66 ; \mathrm{N}, 10.77$.

$\alpha$-Amino- $N$-methyl-1-piperidinepropionamide (8) Methyl $\alpha$-amino1-piperidinepropanoate dihydrochloride $7(2 \mathrm{~g}, 7.7 \mathrm{mmol})$ was added to a $40 \%$ solution of methylamine in methanol $(6 \mathrm{ml})$ and the reaction mixture was allowed to stand at room temperature for $3 \mathrm{~h}$, then the solvent was evaporated to dryness. After addition of $\mathrm{Et}_{2} \mathrm{O}$ to the residue, the separated precipitate was isolated by filtration. The filtrate was concentrated to dryness to give $8(1.06 \mathrm{~g}, 74 \%)$. The precipitate was made basic with small amount of aqueous $\mathrm{NaOH}$ ( $c a .2 \%$ ), extracted with AcOEt, and the extract was concentrated to dryness to give $8(0.18 \mathrm{~g}, 13 \%)$. The total yield was $87 \%$. An analytical sample was obtained by recrystallization from diisopropylether, prisms, mp $99-100^{\circ} \mathrm{C}$. IR (KBr): $3376,3325,1651 \mathrm{~cm}^{-1}$. FAB-MS $m / z$ : $186(\mathrm{M}+\mathrm{H})^{+} .{ }^{1} \mathrm{H}-\mathrm{NMR}\left(\mathrm{CDCl}_{3}\right) \delta: 1.46\left(2 \mathrm{H}, \mathrm{q}, J=6 \mathrm{~Hz}, 4-\mathrm{CH}_{2}\right.$ of piperidine ring), $1.58\left(4 \mathrm{H}, \mathrm{q}, J=6 \mathrm{~Hz}, 3,5-\mathrm{CH}_{2}\right.$ of piperidine ring), $1.95(2 \mathrm{H}, \mathrm{br} \mathrm{s}$, $\left.\mathrm{NH}_{2}\right), 2.36-2.42\left(2 \mathrm{H}, \mathrm{m}, 2-\mathrm{CH}_{2}\right.$ of piperidine ring), 2.39 [1 $\mathrm{H}, \mathrm{dd}, J=13,8$ $\left.\mathrm{Hz}, \mathrm{NH}_{2} \mathrm{CH}(\mathrm{CON}=) \mathrm{C} \underline{\mathrm{H} H N}=\right], 248-2.49\left(2 \mathrm{H}, \mathrm{m}, 6-\mathrm{CH}_{2}\right.$ of piperidine ring), $2.60\left[1 \mathrm{H}, \mathrm{dd}, J=13,6 \mathrm{~Hz}, \mathrm{NH}_{2} \mathrm{CH}(\mathrm{CON}=) \mathrm{CHHN}=\right], 2.80(3 \mathrm{H}, \mathrm{d}$, $\left.J=5 \mathrm{~Hz}, \mathrm{CH}_{3}\right), 3.50\left[1 \mathrm{H}, \mathrm{dd}, J=8,6 \mathrm{~Hz}, \mathrm{NH}_{2} \mathrm{CH}(\mathrm{CON}=) \mathrm{CH}_{2} \mathrm{~N}=\right], 7.88$ (1H, br s, CONH). ${ }^{13} \mathrm{C}-\mathrm{NMR}\left(\mathrm{CDCl}_{3}\right) \delta: 24.2$ (C4 of piperidine ring), 25.7 $\left(\mathrm{NCH}_{3}\right), 26.1(\mathrm{C} 3, \mathrm{C} 5$ of piperidine ring $), 51.6\left[\mathrm{NH}_{2} \mathrm{CH}(\mathrm{CON}=) \mathrm{CH}_{2}-\right], 54.4$ (C2, $\mathrm{C} 6$ of piperidine ring), $62.2\left[\mathrm{NH}_{2} \mathrm{CH}(\mathrm{CON}=) \underline{\mathrm{CH}}_{2}^{-}\right], 175.1$ (CO). Anal. Calcd for $\mathrm{C}_{9} \mathrm{H}_{19} \mathrm{~N}_{3} \mathrm{O}: \mathrm{C}, 58.35 ; \mathrm{H}, 10.34 ; \mathrm{N}, 22.68$. Found: C, 58.32; H, $10.32 ; \mathrm{N}, 22.46$.

2-Amino-3-(1-piperidinyl)-1-(1-pyrrolidinyl)-1-propanone (9) Methyl $\alpha$-amino-1-piperidinepropanoate dihydrochloride $7(2 \mathrm{~g}, 7.7 \mathrm{mmol})$ was added to pyrrolidine $(5.5 \mathrm{ml})$ and stirred for $4 \mathrm{~h} \mathrm{Et}_{2} \mathrm{O}$ was added to the reaction mixture and the precipitate was filtered off. The ethereal solution was concentrated, and the excess pyrrolidine was removed under reduced pressure to give $9(1.31 \mathrm{~g}, 75 \%)$. An analytical sample was obtained by recrystallization from acetone/ $\mathrm{Et}_{2} \mathrm{O} . \mathrm{mp} 42-46^{\circ} \mathrm{C}$. IR $(\mathrm{KBr}): 1638 \mathrm{~cm}^{-1}$. FAB-MS $m / z: 226(\mathrm{M}+\mathrm{H})^{+} .{ }^{1} \mathrm{H}-\mathrm{NMR}\left(\mathrm{CDCl}_{3}\right) \delta: 1.44\left(2 \mathrm{H}\right.$, quintet, $J=6 \mathrm{~Hz}, 4-\mathrm{CH}_{2}$ of piperidine ring), $1.51-1.62\left(4 \mathrm{H}, \mathrm{m}, 3,5-\mathrm{CH}_{2}\right.$ of piperidine ring), 1.86 ( $2 \mathrm{H}$, quintet, $J=7 \mathrm{~Hz}, 3-\mathrm{CH}_{2}$ of pyrrolidine ring $), 1.92-1.99\left(2 \mathrm{H}, \mathrm{m}, 4-\mathrm{CH}_{2}\right.$ of pyrrolidine ring), 2.39 [1 $\mathrm{H}, \mathrm{dd}, J=13,9 \mathrm{~Hz}, \mathrm{NH}_{2} \mathrm{CH}(\mathrm{CON}=) \mathrm{CH} H \mathrm{H}=$ ], $2.43\left(2 \mathrm{H}, \mathrm{m}, 2-\mathrm{CH}_{2}\right.$ of piperidine ring), $2.47(1 \mathrm{H}, \mathrm{dd}, J=13,5 \mathrm{~Hz}$, $\left.\mathrm{NH}_{2} \mathrm{CH}(\mathrm{CON}=) \mathrm{CHHN}=\right), 2.52-2.57\left(2 \mathrm{H}, \mathrm{m}, 6-\mathrm{CH}_{2}\right.$ of piperidine ring $)$, $2.87\left(2 \mathrm{H}, \mathrm{br} \mathrm{s}, \mathrm{NH}_{2}\right), 3.41-3.46\left(2 \mathrm{H}, \mathrm{m}, 2-\mathrm{CH}_{2}\right.$ of pyrrolidine ring $), 3.50-$ $3.58\left(2 \mathrm{H}, \mathrm{m}, 5-\mathrm{CH}_{2}\right.$ of pyrrolidine ring), $3.77(1 \mathrm{H}, \mathrm{dd}, J=9,5 \mathrm{~Hz}$, $\left.\mathrm{NH}_{2} \mathrm{CH}(\mathrm{CON}=) \mathrm{CH}_{2} \mathrm{~N}=\right) \cdot{ }^{13} \mathrm{C}-\mathrm{NMR}\left(\mathrm{CDCl}_{3}\right) \delta: 24.1(\mathrm{C} 4$ of piperidine ring, $\mathrm{C} 3$ of pyrrolidine ring), 25.9 (C3, $\mathrm{C} 5$ of piperidine ring), 26.0 ( $\mathrm{C} 4$ of pyrrolidine ring), 45.9 ( $\mathrm{C} 2$ of pyrrolidine ring), 46.2 (C5 of pyrrolidine ring), 51.0 $\left[\mathrm{NH}_{2} \mathrm{CH}(\mathrm{CON}=) \mathrm{CH}_{2}-\right], \quad 55.1 \quad(\mathrm{C} 2, \quad \mathrm{C} 6$ of piperidine ring), 63.7 $\left[\mathrm{NH}_{2} \mathrm{CH}(\mathrm{CON}=) \mathrm{CH}_{2}-\right], 172.3(\mathrm{CO})$. Anal. Calcd for $\mathrm{C}_{12} \mathrm{H}_{23} \mathrm{~N}_{3} \mathrm{O} \cdot 0.5 \mathrm{H}_{2} \mathrm{O}$ : C, 61.51; H, 10.32; N, 17.93. Found: C, 61.42; H, 10.14; N, 17.72.

$\mathrm{N}$-[1-(Methylaminocarbonyl)-2-(1-piperidinyl)ethyl]benzamide (4a) A solution of DCC $(1.34 \mathrm{~g}, 6.5 \mathrm{mmol})$ in $\mathrm{CH}_{2} \mathrm{Cl}_{2}$ was added to a stirred solution of $8(1.0 \mathrm{~g}, 5.4 \mathrm{mmol})$ and benzoic acid $(0.79 \mathrm{~g}, 6.5 \mathrm{mmol})$ in $\mathrm{CH}_{2} \mathrm{Cl}_{2}$ and the mixture was stirred at room temperature for $1 \mathrm{~d}$. The dicyclohexylurea (DCU) was removed by filtration and washed with a small amount of
$\mathrm{CH}_{2} \mathrm{Cl}_{2}$. The filtrate was extracted three times with $1 \mathrm{~N} \mathrm{HCl}$ and the extract was washed with $\mathrm{CH}_{2} \mathrm{Cl}_{2}$. The aqueous solution was made basic with anhydrous $\mathrm{K}_{2} \mathrm{CO}_{3}$ and the resulting precipitate was extracted with $\mathrm{CH}_{2} \mathrm{Cl}_{2}$. The extract was washed with brine, dried over anhydrous $\mathrm{MgSO}_{4}$, and concentrated in vacuo to give $4 \mathrm{a}(0.76 \mathrm{~g}, 49 \%)$ which was recrystallized from AcOEt to afford colorless prisms. IR (KBr): $3315,3265,1674,1630 \mathrm{~cm}^{-1}$. ${ }^{1} \mathrm{H}-\mathrm{NMR}\left(\mathrm{CDCl}_{3}\right) \delta: 1.47-1.50\left(2 \mathrm{H}, \mathrm{m}, 4-\mathrm{CH}_{2}\right.$ of piperidine ring), $1.57-$ $1.62\left(4 \mathrm{H}, \mathrm{m}, 3,5-\mathrm{CH}_{2}\right.$ of piperidine ring), $2.42-2.57\left(2 \mathrm{H}, \mathrm{m}, 2-\mathrm{CH}_{2}\right.$ of piperidine ring), $2.47[1 \mathrm{H}, \mathrm{dd}, J=13,10 \mathrm{~Hz},-\mathrm{NHCH}(\mathrm{CON}=) \mathrm{C} \underline{\mathrm{HHN}}=]$, $2.75\left(2 \mathrm{H}, \mathrm{br}, 6-\mathrm{CH}_{2}\right.$ of piperidine ring), $2.84\left(3 \mathrm{H}, \mathrm{d}, J=5 \mathrm{~Hz}, \mathrm{NCH}_{3}\right), 2.89$ $[1 \mathrm{H}, \mathrm{dd}, J=13,5 \mathrm{~Hz},-\mathrm{NHCH}(\mathrm{CON}=) \mathrm{CH} \underline{\mathrm{HN}}=], 4.51[1 \mathrm{H}, \mathrm{dt}, J=10,5 \mathrm{~Hz}$, $\left.\mathrm{m},-\mathrm{NHC} \underline{\mathrm{H}}(\mathrm{CON}=) \mathrm{CH}_{2} \mathrm{~N}=\right], 7.40-7.44(2 \mathrm{H}, \mathrm{m}, 3,5 \mathrm{H}$-aromatic $), 7.47-$ $7.50(2 \mathrm{H}, \mathrm{m}, 4 \mathrm{H}$-aromatic, $\mathrm{Ar}-\mathrm{CON} \underline{\mathrm{H}}), 7.82-7.84(2 \mathrm{H}, \mathrm{m}, 2,6 \mathrm{H}$-aromatic), $8.25\left(1 \mathrm{H}\right.$, brd, $\left.J=5 \mathrm{~Hz}, \mathrm{NHCOCH}_{3}\right) .{ }^{13} \mathrm{C}-\mathrm{NMR}\left(\mathrm{CDCl}_{3}\right) \delta: 23.9(\mathrm{C} 4$ of piperidine ring), $25.9\left(\mathrm{NCH}_{3}\right), 26.2$ (C3, $\mathrm{C} 5$ of piperidine ring), 49.5 $\left[-\mathrm{NH} \underline{\mathrm{CH}}(\mathrm{CON}=) \mathrm{CH}_{2}-\right], \quad 54.3 \quad(\mathrm{C} 2, \quad \mathrm{C} 6$ of piperidine ring $), \quad 59.6$ $\left[-\mathrm{NHCH}(\mathrm{CON}=) \mathrm{CH}_{2}-\right], 127.0(\mathrm{C} 2, \mathrm{C} 6$-aromatic), 128.4 (C3, C5-aromatic), 131.5 (C4-aromatic), 139.9 (C1-aromatic), 167.1 (Ar-으), 171.9 (ㅁN$\mathrm{HCH}_{3}$ ).

Compounds $\mathbf{4 b}-\mathbf{d}$ and $\mathbf{5 a}-\mathbf{d}$ were also obtained according to the above procedure.

4-Amino-3-chloro-6-methoxy- $N$-[1-(methylaminocarbonyl)-2-(1piperidinyl)ethyl]benzamide (4b): The yield was $35 \%$. Treatment of $\mathbf{4 b}$ with $20 \% \mathrm{HCl} / \mathrm{EtOH}$ gave the hydrochloride of $\mathbf{4 b}$. IR $(\mathrm{KBr}): 3465,1684,1622$, $1606 \mathrm{~cm}^{-1}$. ${ }^{1} \mathrm{H}-\mathrm{NMR}\left(\mathrm{D}_{2} \mathrm{O}\right) \delta: 1.67\left(2 \mathrm{H}, \mathrm{brs}, 4-\mathrm{CH}_{2}\right.$ of piperidine ring), $1.87-1.88\left(4 \mathrm{H}, \mathrm{m}, 3,5-\mathrm{CH}_{2}\right.$ of piperidine ring $), 2.82\left(3 \mathrm{H}, \mathrm{s}, \mathrm{NCH}_{3}\right), 3.30-$ $3.38\left(4 \mathrm{H}, \mathrm{m}, 2,6-\mathrm{CH}_{2}\right.$ of piperidine ring), $3.45[1 \mathrm{H}, \mathrm{dd}, J=14$, $9 \mathrm{~Hz},-\mathrm{NHCH}(\mathrm{CON}=) \mathrm{CHHN}=], 3.68[1 \mathrm{H}, \mathrm{dd}, J=14,5 \mathrm{~Hz},-\mathrm{NHCH}$ $(\mathrm{CON}=) \mathrm{CHHN}=], 3.95\left(3 \mathrm{H}, \mathrm{s}, \mathrm{OCH}_{3}\right), 5.07 \quad[1 \mathrm{H}, \mathrm{dd}, J=9,5 \mathrm{~Hz}$, $-\mathrm{NHCH}(\mathrm{CON}=) \mathrm{CH}_{2} \mathrm{~N}=$ ], $6.57(1 \mathrm{H}, \mathrm{s}, 3 \mathrm{H}$-aromatic $), 7.76(1 \mathrm{H}, \mathrm{s}, 6 \mathrm{H}$-aromatic) ${ }^{13} \mathrm{C}$-NMR $\left(\mathrm{D}_{2} \mathrm{O}\right) \delta: 23.8$ (C4 of piperidine ring), $25.6(\mathrm{C} 3, \mathrm{C} 5$ of piperidine ring), $29.0\left(\mathrm{NCH}_{3}\right), 52.0\left[-\mathrm{NH} \underline{\mathrm{CH}}(\mathrm{CON}=) \mathrm{CH}_{2}-\right], 57.0(\mathrm{C} 2, \mathrm{C} 6$ of piperidine ring $), 59.1\left(\mathrm{OCH}_{3}\right), 60.7\left[-\mathrm{NHCH}(\mathrm{CON}=) \mathrm{CH}_{2}-\right], 101.5(\mathrm{C} 3-$ aromatic), 112.4 (C1-aromatic), 114.0 (C5-aromatic), 134.6 (C6-aromatic), 151.9 (C4-aromatic), 161.2 (C2-aromatic), $170.1\left(\mathrm{CONHCH}_{3}\right), 173.2(\mathrm{Ar}-$ $\underline{\mathrm{CO}}$.

3,4-Dimethoxy- $N$-[1-(methylaminocarbonyl)-2-(1-piperidinyl)ethyl]benzamide (4c): The yield was $26 \%$. IR ( $\mathrm{KBr}): 3260,1620 \mathrm{~cm}^{-1}$. ${ }^{1} \mathrm{H}-\mathrm{NMR}$ $\left(\mathrm{CDCl}_{3}\right) \delta: 1.48-1.52\left(2 \mathrm{H}, \mathrm{m}, 4-\mathrm{CH}_{2}\right.$ of piperidine ring $), 1.59-1.64(4 \mathrm{H}$, $\mathrm{m}, 3,5-\mathrm{CH}_{2}$ of piperidine ring), $2.44-2.48\left[3 \mathrm{H}, \mathrm{m}, 2-\mathrm{CH}_{2}\right.$ of piperidine ring, $-\mathrm{NHCH}(\mathrm{CON}=) \mathrm{CHHN}=], 2.77\left(2 \mathrm{H}, \mathrm{br}, 6-\mathrm{CH}_{2}\right.$ of piperidine ring $)$, $2.86\left(3 \mathrm{H}, \quad \mathrm{d}, \quad J=5 \mathrm{~Hz}, \quad \mathrm{NCH}_{3}\right), 2.91 \quad[1 \mathrm{H}, \quad \mathrm{dd}, \quad J=13, \quad 4 \mathrm{~Hz}$, $-\mathrm{NHCH}(\mathrm{CON}=) \mathrm{CH} \underline{\mathrm{HN}}=], 3.921,3.922\left(\right.$ each $\left.3 \mathrm{H}, \mathrm{s}, \mathrm{OCH}_{3}\right), 4.46-4.49$ $\left[1 \mathrm{H}, \mathrm{m},-\mathrm{NHCH}(\mathrm{CON}=) \mathrm{CH}_{2} \mathrm{~N}=\right], 6.88(1 \mathrm{H}, \mathrm{d}, J=8 \mathrm{~Hz}, 5 \mathrm{H}$-aromatic), $7.35-7.45(1 \mathrm{H}, \mathrm{br}, \mathrm{Ar}-\mathrm{CON} \underline{\mathrm{H}}), 7.39(1 \mathrm{H}, \mathrm{dd}, J=8,2 \mathrm{~Hz}, 6 \mathrm{H}$-aromatic), $7.45\left(1 \mathrm{H}, \mathrm{d}, J=2 \mathrm{~Hz}, 2 \mathrm{H}\right.$-aromatic), $8.26\left(1 \mathrm{H}, \mathrm{br} \mathrm{s}, \mathrm{CONHCH}_{3}\right) \cdot{ }^{13} \mathrm{C}-\mathrm{NMR}$ $\left(\mathrm{CDCl}_{3}\right) \delta: 24.0$ (C4 of piperidine ring), $26.0\left(\mathrm{NCH}_{3}\right), 26.3(\mathrm{C} 3, \mathrm{C} 5$ of piperidine ring), $49.6\left[-\mathrm{NHCH}(\mathrm{CON}=) \mathrm{CH}_{2}-\right], 54.4(\mathrm{C} 2, \mathrm{C} 6$ of piperidine ring), $56.0\left(\mathrm{OCH}_{3} \times 2\right), 59.7\left[-\mathrm{NHCH}(\mathrm{CON}=) \mathrm{CH}_{2}-\right], 110.4(\mathrm{C} 2$ or $\mathrm{C} 5$-aromatic), 110.5 (C5 or C2-aromatic), 119.8 (C6-aromatic), 126.6 (C1-aromatic), 149.0 (C3-aromatic), 152.0 (C4-aromatic), 166.8 (Ar-O), 172.2 $\left(\mathrm{CONHCH}_{3}\right)$.

3,4,5-Trimethoxy- $N$-[1-(methylaminocarbonyl)-2-(1-piperidinyl)ethyl]benzamide (4d): The yield was $33 \%$. IR (KBr): $3281,1662,1622 \mathrm{~cm}^{-1}$. ${ }^{1} \mathrm{H}-\mathrm{NMR}\left(\mathrm{CDCl}_{3}\right) \delta: 1.49-1.50\left(2 \mathrm{H}, \mathrm{m}, 4-\mathrm{CH}_{2}\right.$ of piperidine ring), $1.51-$ $1.52\left(4 \mathrm{H}, \mathrm{m}, 3,5-\mathrm{CH}_{2}\right.$ of piperidine ring), $2.45-2.49\left[3 \mathrm{H}, \mathrm{m}, 2-\mathrm{CH}_{2}\right.$ of piperidine ring, $-\mathrm{NHCH}(\mathrm{CON}=) \mathrm{CHHN}=], 2.79\left(2 \mathrm{H}, \mathrm{m}, 6-\mathrm{CH}_{2}\right.$ of piperidine ring), $2.86\left(3 \mathrm{H}, \quad \mathrm{d}, J=4.5 \mathrm{~Hz}, \mathrm{NCH}_{3}\right), 2.91-2.93[1 \mathrm{H}, \mathrm{m}$, $-\mathrm{NHCH}(\mathrm{CON}=) \mathrm{CH} \underline{\mathrm{HN}}=], 3.88\left(3 \mathrm{H}, \mathrm{s}, 4^{\prime}-\mathrm{OCH}_{3}\right), 3.90\left(6 \mathrm{H}, \mathrm{s}, 3^{\prime}, 5^{\prime}-\right.$ $\left.\mathrm{OCH}_{3}\right), 4.46-4.49\left[1 \mathrm{H}, \mathrm{m},-\mathrm{NHCH}(\mathrm{CON}=) \mathrm{CH}_{2} \mathrm{~N}=\right], 7.09(2 \mathrm{H}, \mathrm{s}, 2,6 \mathrm{H}-$ aromatic), $7.27(1 \mathrm{H}, \mathrm{br}, \mathrm{Ar}-\mathrm{CON} \underline{\mathrm{H}}), 8.30\left(1 \mathrm{H}, \mathrm{br}, \mathrm{CONHCH}_{3}\right) .{ }^{13} \mathrm{C}-\mathrm{NMR}$ $\left(\mathrm{CDCl}_{3}\right) \delta$ : 24.0 (C4 of piperidine ring), $26.0\left(\mathrm{NCH}_{3}\right), 26.3(\mathrm{C} 3, \mathrm{C} 5$ of piperidine ring $), 50.0\left[-\mathrm{NH} \underline{\mathrm{CH}}(\mathrm{CON}=) \mathrm{CH}_{2}-\right], 54.4(\mathrm{C} 2, \mathrm{C} 6$ of piperidine ring), $56.3\left(3^{\prime}, 5^{\prime}-\mathrm{OCH}_{3}\right), 59.6\left[-\mathrm{NHCH}(\mathrm{CON}=) \underline{\mathrm{CH}}_{2}-\right], 60.9\left(4^{\prime}-\mathrm{OCH}_{3}\right)$, 104.5, (C2, C6-aromatic), 129.2 (C1-aromatic), 141.3 (C4-aromatic), 153.2 (C3, C5-aromatic), 166.8 (Ar- $\underline{-} \mathrm{O}), 172.1\left(\mathrm{CONHCH}_{3}\right)$.

$N$-[2-(1-Piperidinyl)-1-(1-pyrrolidinylcarbonyl)ethyl]benzamide (5a): The yield was $74 \%$. Treatment of $\mathbf{5 a}$ with $20 \% \mathrm{HCl} / \mathrm{EtOH}$ gave the hydrochloride of $\mathbf{5 a}$, which was recrystallized from $\mathrm{CH}_{3} \mathrm{CN}$ to afford colorless needles. IR (KBr): 3218, 1666, $1645 \mathrm{~cm}^{-1} .{ }^{1} \mathrm{H}-\mathrm{NMR}\left(\mathrm{D}_{2} \mathrm{O}\right) \delta: 1.68$ $\left(2 \mathrm{H}\right.$, br s, $4-\mathrm{CH}_{2}$ of piperidine ring), $1.85-1.91\left(4 \mathrm{H}, \mathrm{m}, 3,5-\mathrm{CH}_{2}\right.$ of piperidine ring), $1.93-2.01\left(4 \mathrm{H}, \mathrm{m}, 3,4-\mathrm{CH}_{2}\right.$ of pyrrolidine ring), $3.33-$ $3.61\left(4 \mathrm{H}, \mathrm{m}, 2,6-\mathrm{CH}_{2}\right.$ of piperidine ring), $3.48-3.67\left[6 \mathrm{H}, \mathrm{m}, 2,5-\mathrm{CH}_{2}\right.$ of pyrrolidine ring, $\left.-\mathrm{NHCH}(\mathrm{CON}=) \mathrm{CH}_{2} \mathrm{~N}=\right], \quad 5.30-5.33 \quad[1 \mathrm{H}, \quad \mathrm{m}$, 
$\left.-\mathrm{NHC} \underline{\mathrm{H}}(\mathrm{CON}=) \mathrm{CH}_{2} \mathrm{~N}=\right], 7.56-7.60(2 \mathrm{H}, \mathrm{m}, 3,5 \mathrm{H}$-aromatic $), 7.67-7.71$ (1H, m, 4H-aromatic), 7.85-7.88 (2H, m, 2,6H-aromatic). ${ }^{13} \mathrm{C}-\mathrm{NMR}\left(\mathrm{D}_{2} \mathrm{O}\right)$ $\delta: 23.8$ ( $\mathrm{C} 4$ of piperidine ring), 25.4 ( $\mathrm{C} 3, \mathrm{C} 5$ of piperidine ring), 26.4 (C3 of pyrrolidine ring), 28.3 ( $\mathrm{C} 4$ of pyrrolidine ring), 49.7 ( $\mathrm{C} 2$ of pyrrolidine ring), 50.0 ( $\mathrm{C} 5$ of pyrrolidine ring), $50.6\left[-\mathrm{NH} \underline{\mathrm{CH}}(\mathrm{CON}=) \mathrm{CH}_{2}-\right], 57.1(\mathrm{C} 2$, $\mathrm{C} 6$ of piperidine ring), $59.6\left[-\mathrm{NHCH}(\mathrm{CON}=) \mathrm{CH}_{2}-\right], 130.2$ (C2, C6-aromatic), 131.8 (C3, C5-aromatic), 134.8 (C1-aromatic), 135.8 (C4-aromatic), $169.9(=\mathrm{CH} \underline{\mathrm{CON}}=)$ ), $173.3(\mathrm{Ar}-\underline{\mathrm{CO}})$. This compound 5a could also be prepared in a similar manner described for the preparation of $5 \mathbf{e}$ in $63 \%$ yield.

4-Amino-3-chloro-6-methoxy- $N$-[2-(1-piperidinyl)-1-(1-pyrrolidinylcarbonyl)ethyl]benzamide (5b): The yield was 35\%. IR (KBr): 3368 , $1638 \mathrm{~cm}^{-1} .{ }^{1} \mathrm{H}-\mathrm{NMR}\left(\mathrm{CDCl}_{3}\right) \delta: 1.40-1.42\left(2 \mathrm{H}, \mathrm{m}, 4-\mathrm{CH}_{2}\right.$ of piperidine ring), $1.50-1.55\left(4 \mathrm{H}, \mathrm{m}, 3,5-\mathrm{CH}_{2}\right.$ of piperidine ring), $1.86-1.90$ $\left(2 \mathrm{H}, \mathrm{m}, 3-\mathrm{CH}_{2}\right.$ of pyrrolidine ring $), 1.95-2.00\left(2 \mathrm{H}, \mathrm{m}, 4-\mathrm{CH}_{2}\right.$ of pyrrolidine ring), $2.47\left(4 \mathrm{H}, \mathrm{d}, J=5 \mathrm{~Hz}, 2,6-\mathrm{CH}_{2}\right.$ of piperidine ring), $2.62[1 \mathrm{H}$, dd, $J=13,7 \mathrm{~Hz},-\mathrm{NHCH}(\mathrm{CON}=) \mathrm{CHHN}=], 2.70[1 \mathrm{H}, \mathrm{dd}, J=13,7 \mathrm{~Hz}$, $-\mathrm{NHCH}(\mathrm{CON}=) \mathrm{CH} \underline{\mathrm{HN}}=], 3.43-3.47\left(1 \mathrm{H}, \mathrm{m}, 2-\mathrm{CH}_{\mathrm{A}}\right.$ of pyrrolidine ring $)$, $3.54-3.57\left(1 \mathrm{H}, \mathrm{m}, 2-\mathrm{CH}_{\mathrm{B}}\right.$ of pyrrolidine ring $), 3.72-3.76\left(2 \mathrm{H}, \mathrm{m}, 5-\mathrm{CH}_{2}\right.$ of pyrrolidine ring), $3.84\left(3 \mathrm{H}, \mathrm{s}, \mathrm{OCH}_{3}\right), 4.57\left(2 \mathrm{H}, \mathrm{s}, \mathrm{Ar}-\mathrm{N}_{2}\right), 4.93-4.94$ $\left[1 \mathrm{H}, \mathrm{q}, J=7 \mathrm{~Hz},-\mathrm{NHCH}(\mathrm{CON}=) \mathrm{CH}_{2} \mathrm{~N}=\right], 6.23(1 \mathrm{H}, \mathrm{s}, 3 \mathrm{H}$-aromatic $), 8.03$ $\left(1 \mathrm{H}, \mathrm{s}, 6 \mathrm{H}\right.$-aromatic), $8.48(1 \mathrm{H}, \mathrm{brd}, J=7 \mathrm{~Hz}, \mathrm{CONH}) .{ }^{13} \mathrm{C}-\mathrm{NMR}\left(\mathrm{CDCl}_{3}\right) \delta$ : 24.2 ( $\mathrm{C} 4$ of piperidine ring), 26.0 ( $\mathrm{C} 3, \mathrm{C} 4$ of pyrrolidine ring), 26.2 (C3, C5 of piperidine ring), 46.0 ( $\mathrm{C} 2$ of pyrrolidine ring), 46.7 ( $\mathrm{C} 5$ of pyrrolidine ring), $49.9\left[-\mathrm{NHCH}(\mathrm{CON}=) \mathrm{CH}_{2}-\right], 55.0(\mathrm{C} 2, \mathrm{C} 6$ of piperidine ring $), 56.0$ $\left(\mathrm{OCH}_{3}\right), 61.0\left[-\mathrm{NHCH}(\mathrm{CON}=) \mathrm{CH}_{2}-\right], 97.8$ (C3-aromatic), $111.3(\mathrm{C} 1-$ aromatic), 112.1 (C5-aromatic), 132.8 (C6-aromatic), 147.0 (C4-aromatic), $157.8(\mathrm{C} 2$-aromatic), $164.0(\mathrm{Ar}-\underline{\mathrm{CO}}), 170.6(=\mathrm{CHCON}=)$.

3,4-Dimethoxy- $N$-[2-(1-piperidinyl)-1-(1-pyrrolidinylcarbonyl)ethyl]benzamide (5c): The yield was $45 \%$. IR ( $\mathrm{KBr}): 3245,1628 \mathrm{~cm}^{-1}$. ${ }^{1} \mathrm{H}-\mathrm{NMR}$ $\left(\mathrm{CDCl}_{3}\right) \delta: 1.40-1.43\left(2 \mathrm{H}, \mathrm{m}, 4-\mathrm{CH}_{2}\right.$ of piperidine ring $), 1.51-1.55(4 \mathrm{H}, \mathrm{m}$, $3,5-\mathrm{CH}_{2}$ of piperidine ring), $1.88-1.92\left(2 \mathrm{H}, \mathrm{m}, 3-\mathrm{CH}_{2}\right.$ of pyrrolidine ring), $1.96-2.04\left(2 \mathrm{H}, \mathrm{m}, 4-\mathrm{CH}_{2}\right.$ of pyrrolidine ring), $2.46-2.51\left(4 \mathrm{H}, \mathrm{m}, 2,6-\mathrm{CH}_{2}\right.$ of piperidine ring), $2.66[1 \mathrm{H}, \mathrm{dd}, J=13,7 \mathrm{~Hz},-\mathrm{NHCH}(\mathrm{CON}=) \mathrm{CHHN}=]$, $2.73[1 \mathrm{H}, \mathrm{dd}, J=13,7 \mathrm{~Hz},-\mathrm{NHCH}(\mathrm{CON}=) \mathrm{CH} \underline{\mathrm{HN}}=], 3.45-3.49(1 \mathrm{H}, \mathrm{m}$, $2-\mathrm{CH}_{\mathrm{A}}$ of pyrrolidine ring), $3.53-3.58\left(1 \mathrm{H}, \mathrm{m}, 2-\mathrm{CH}_{\mathrm{B}}\right.$ of pyrrolidine ring), $3.75-3.78\left(2 \mathrm{H}, \mathrm{m}, 5-\mathrm{CH}_{2}\right.$ of pyrrolidine ring), 3.91, 3.92 (each $3 \mathrm{H}, \mathrm{s}$, $\left.\mathrm{OCH}_{3}\right), 4.97\left[1 \mathrm{H}, \mathrm{q}, J=7 \mathrm{~Hz},-\mathrm{NHCH}(\mathrm{CON}=) \mathrm{CH}_{2} \mathrm{~N}=\right.$ ], $6.86(1 \mathrm{H}, \mathrm{d}, J=8$ $\mathrm{Hz}, 5 \mathrm{H}$-aromatic), $7.08(1 \mathrm{H}$, br d, $J=7 \mathrm{~Hz}, \mathrm{CONH}), 7.36(1 \mathrm{H}, \mathrm{dd}, J=8,2 \mathrm{~Hz}$, $6 \mathrm{H}$-aromatic), $7.43\left(1 \mathrm{H}, \mathrm{d}, J=2 \mathrm{~Hz}, 2 \mathrm{H}\right.$-aromatic). ${ }^{13} \mathrm{C}-\mathrm{NMR}\left(\mathrm{CDCl}_{3}\right) \delta$ : 24.2 (C4 of piperidine ring), 26.0 (C3, C4 of pyrrolidine ring), 26.2 (C3, C5 of piperidine ring), 46.0 (C2 of pyrrolidine ring), 46.7 (C5 of pyrrolidine ring), $49.6\left[-\mathrm{NHCH}(\mathrm{CON}=) \mathrm{CH}_{2}-\right], 55.0(\mathrm{C} 2, \mathrm{C} 6$ of piperidine ring), 56.0 $\left(\mathrm{OCH}_{3} \times 2\right), 61.0\left[-\mathrm{NHCH}(\mathrm{CON}=) \mathrm{CH}_{2}-\right], 110.3(\mathrm{C} 2$ or $\mathrm{C} 5$-aromatic $), 110.6$ (C5 or C2-aromatic), 120.0 (C6-aromatic), 126.7 (C1-aromatic), 148.9 (C3aromatic), 151.8 (C4-aromatic), $166.3(\mathrm{Ar}-\underline{\mathrm{CO}}), 170.3(=\mathrm{CHCON}=)$.

3,4,5-Trimethoxy- $N$-[2-(1-piperidinyl)-1-(1-pyrrolidinylcarbonyl)ethyl]benzamide (5d): The yield was $50 \%$. IR $(\mathrm{KBr}): 3318,1664,1633 \mathrm{~cm}^{-1}$. ${ }^{1} \mathrm{H}-\mathrm{NMR} \quad\left(\mathrm{CDCl}_{3}\right) \quad \delta: \quad 1.40-1.44 \quad\left(2 \mathrm{H}, \quad \mathrm{m}, \quad 4-\mathrm{CH}_{2}\right.$ of piperidine ring), $1.51-1.55\left(4 \mathrm{H}, \mathrm{m}, 3,5-\mathrm{CH}_{2}\right.$ of piperidine ring $), 1.88-1.92(2 \mathrm{H}$, $\mathrm{m}, 3-\mathrm{CH}_{2}$ of pyrrolidine ring $), 1.98-2.02\left(2 \mathrm{H}, \mathrm{m}, 4-\mathrm{CH}_{2}\right.$ of pyrrolidine ring), $2.46-2.52\left(4 \mathrm{H}, \mathrm{m}, 2,6-\mathrm{CH}_{2}\right.$ of piperidine ring $), 2.66[1 \mathrm{H}, \mathrm{dd}$, $J=13,7 \mathrm{~Hz},-\mathrm{NHCH}(\mathrm{CON}=) \mathrm{CHHN}=], 2.74 \quad[1 \mathrm{H}, \mathrm{dd}, J=13,7 \mathrm{~Hz}$, $-\mathrm{NHCH}(\mathrm{CON}=) \mathrm{CH} \underline{\mathrm{HN}}=], 3.45-3.49\left(1 \mathrm{H}, \mathrm{m}, 2-\mathrm{CH}_{\mathrm{A}}\right.$ of pyrrolidine ring $)$, $3.53-3.56\left(1 \mathrm{H}, \mathrm{m}, 2-\mathrm{CH}_{\mathrm{B}}\right.$ of pyrrolidine ring $), 3.76\left(2 \mathrm{H}, \mathrm{t}, J=7 \mathrm{~Hz}, 5-\mathrm{CH}_{2}\right.$ of pyrrolidine ring), $3.87\left(3 \mathrm{H}, \mathrm{s}, 4^{\prime}-\mathrm{OCH}_{3}\right), 3.89\left(6 \mathrm{H}, \mathrm{s}, 3^{\prime}, 5^{\prime}-\mathrm{OCH}_{3}\right), 4.96$ $\left[1 \mathrm{H}, \mathrm{q}, J=7 \mathrm{~Hz},-\mathrm{NHC}(\mathrm{CON}=) \mathrm{CH}_{2} \mathrm{~N}=\right], 7.06(2 \mathrm{H}, \mathrm{s}, 2,6 \mathrm{H}$-aromatic), $7.15(1 \mathrm{H}, \mathrm{d}, J=7 \mathrm{~Hz}, \mathrm{CONH}) .{ }^{13} \mathrm{C}-\mathrm{NMR}\left(\mathrm{CDCl}_{3}\right) \delta: 24.2(\mathrm{C} 4$ of piperidine ring), 26.0 ( $\mathrm{C} 3, \mathrm{C} 4$ of pyrrolidine ring), 26.2 ( $\mathrm{C} 3, \mathrm{C} 5$ of piperidine ring), 46.0 (C2 of pyrrolidine ring), 46.7 ( $\mathrm{C} 5$ of pyrrolidine ring), 49.7 $\left[-\mathrm{NHCH}(\mathrm{CON}=) \mathrm{CH}_{2}-\right], 54.9\left(\mathrm{C} 2, \mathrm{C} 6\right.$ of piperidine ring), $56.3\left(3^{\prime}, 5^{\prime}-\right.$ $\left.\mathrm{OCH}_{3}\right), 60.8\left[-\mathrm{NHCH}(\mathrm{CON}=) \mathrm{CH}_{2}-, 4^{\prime}-\mathrm{OCH}_{3}\right], 104.6(\mathrm{C} 2, \mathrm{C} 6$-aromatic $)$, 129.4 (C1-aromatic), 141.1 (C4-aromatic), 153.1 (C3, C5-aromatic), 166.3 $(\mathrm{Ar}-\underline{\mathrm{CO}}), 170.2(=\mathrm{CHCON}=)$.

$\alpha$-(Benzoylamino)-1-piperidinepropanoic Acid (10) Using a threeneck round-bottomed flask, benzoyl chloride $(8.03 \mathrm{~g}, 57 \mathrm{mmol})$ was added dropwise to a vigorously stirred solution of $6(7 \mathrm{~g}, 28.6 \mathrm{mmol})$ in $1 \mathrm{~N} \mathrm{NaOH}(86 \mathrm{ml})$ and $2 \mathrm{~N} \mathrm{NaOH}(43 \mathrm{ml})$ was added at the same time. In this experiment, it is advantageous to terminate the addition of both reagents together. The reaction mixture was stirred for $2 \mathrm{~h}$ at room temperature and then acidified with $20 \% \mathrm{HCl}$. The resulting mixture was washed with diethyl ether $\left(\mathrm{Et}_{2} \mathrm{O}\right)$ and the resulting aqueous layer was evaporated to give residual oil. After the addition of ethanol to this residue, the precipitated insoluble solid was removed by filtration. The mother liquor was concentrated to dryness. The residue was passed through a column packed with ion-exchange resin (AG 11A $8^{\circledR}$, Bio-Rad Laboratories) to give crude product, which was recrystallized from $\mathrm{H}_{2} \mathrm{O}$ to afford 10 (5.3 g, 67\%), mp $139-141^{\circ} \mathrm{C}$ (dec.). IR (KBr): 1647, 1605 $\mathrm{cm}^{-1}$. FAB-MS $\mathrm{m} / z: 277(\mathrm{M}+\mathrm{H})^{+} .{ }^{1} \mathrm{H}-\mathrm{NMR}\left(\mathrm{D}_{2} \mathrm{O}\right) \delta: 1.55-2.10(6 \mathrm{H}$, br, 3,4,5- $\mathrm{CH}_{2}$ of piperidine ring), $2.90-3.82\left(4 \mathrm{H}, \mathrm{br}, 2,6-\mathrm{CH}_{2}\right.$ of piperidine ring $), 3.47[1 \mathrm{H}, \mathrm{dd}, J=13,8 \mathrm{~Hz},-\mathrm{NHCH}(\mathrm{CON}=) \mathrm{CHHN}=], 3.61[1 \mathrm{H}$, $\mathrm{dd}, J=13,6 \mathrm{~Hz},-\mathrm{NHCH}(\mathrm{CON}=) \mathrm{CH} \underline{\mathrm{HN}}=], 4.84[1 \mathrm{H}, \mathrm{dd}, J=8,6 \mathrm{~Hz}$, $\left.-\mathrm{NHCH}(\mathrm{CON}=) \mathrm{CH}_{2} \mathrm{~N}=\right], 7.57(2 \mathrm{H}, \mathrm{t}, J=8 \mathrm{~Hz}, 3,5 \mathrm{H}$-aromatic $), 7.67(1 \mathrm{H}$, $\mathrm{t}, J=8 \mathrm{~Hz}, 4 \mathrm{H}$-aromatic), $7.86\left(2 \mathrm{H}\right.$, d-like, $J=8 \mathrm{~Hz}, 2,6 \mathrm{H}$-aromatic). ${ }^{13} \mathrm{C}-$ NMR $\left(\mathrm{D}_{2} \mathrm{O}\right) \delta: 23.9(\mathrm{C} 4$ of piperidine ring), $25.6(\mathrm{C} 3, \mathrm{C} 5$ of piperidine ring), $52.8\left[-\mathrm{NH} \underline{\mathrm{C}} \mathrm{H}(\mathrm{CON}=) \mathrm{CH}_{2}-\right], 56.6(\mathrm{C} 2, \mathrm{C} 6$ of piperidine ring), 60.9 $\left[-\mathrm{NHCH}(\mathrm{CON}=) \mathrm{CH}_{2}-\right], 130.2$ (C2, C6-aromatic), 131.7 (C3, C5-aromatic), 135.5 (C1 or C4-aromatic), 135.6 (C4 or $\mathrm{C} 1$-aromatic), $173.4(\mathrm{CONH})$, $176.8(\mathrm{COOH})$. Anal. Calcd for $\mathrm{C}_{15} \mathrm{H}_{20} \mathrm{~N}_{2} \mathrm{O}_{3} \cdot \mathrm{H}_{2} \mathrm{O}: \mathrm{C}, 61.21 ; \mathrm{H}, 7.53 ; \mathrm{N}$, 9.52. Found: C, $61.08 ; \mathrm{H}, 7.53 ; \mathrm{N}, 9.51$.

$\mathrm{N}$-[2-(1-Piperidinyl)-1-(1-piperidinylcarbonyl)ethyl]benzamide (5e) (Method via Mixed Anhydride) To a solution of compound $\mathbf{1 0}$ ( $1 \mathrm{~g}, 3.6$ $\mathrm{mmol})$ and $N$-methylmorpholine $(0.56 \mathrm{~g}, 5.5 \mathrm{mmol})$ in anhydrous dimethylformamide (DMF) $(50 \mathrm{ml})$, pivaloyl chloride $(0.67 \mathrm{~g}, 5.5 \mathrm{mmol})$ was added at $-15^{\circ} \mathrm{C}$ with stirring. After $5 \mathrm{~min}$, piperidine $(0.47 \mathrm{~g}, 5.5 \mathrm{mmol})$ was added and the reaction mixture was allowed to stand at $0{ }^{\circ} \mathrm{C}$ for $30 \mathrm{~min}$ and then overnight at room temperature with stirring. The reaction mixture was concentrated in vacuo, the residue was extracted with $1 \mathrm{~N} \mathrm{HCl}$, and the aqueous solution was washed with $\mathrm{Et}_{2} \mathrm{O}$ and basified with $\mathrm{K}_{2} \mathrm{CO}_{3}$. The resulting precipitate was collected by filtration, washed with water, and dried to give $\mathbf{5 e}$ $(0.62 \mathrm{~g}, 50 \%)$, which was recrystallized from $\mathrm{CH}_{3} \mathrm{CN}$ to afford colorless needles. IR (KBr): $3300,1653,1619 \mathrm{~cm}^{-1}$. ${ }^{1} \mathrm{H}-\mathrm{NMR}\left(\mathrm{CDCl}_{3}\right) \delta: 1.39-1.43$ $\left(2 \mathrm{H}, \mathrm{m}, 4-\mathrm{CH}_{2}\right.$ of piperidine ring $), 1.51-1.59\left(6 \mathrm{H}, \mathrm{m}, 3,5-\mathrm{CH}_{2}\right.$ of piperidine ring, $4-\mathrm{CH}_{2}$ of 1-acylpiperidine ring), $1.67\left(4 \mathrm{H}\right.$, br s, 3,5- $\mathrm{CH}_{2}$ of 1acylpiperidine ring), $2.44-2.56\left(4 \mathrm{H}, \mathrm{m}, 2,6-\mathrm{CH}_{2}\right.$ of piperidine ring), 2.64 $2.69\left[2 \mathrm{H}, \mathrm{m},-\mathrm{NHCH}(\mathrm{CON}=) \mathrm{CH}_{2} \mathrm{~N}=\right], 3.53-3.58\left(2 \mathrm{H}, \mathrm{m}, 2,6-\mathrm{CH}_{\mathrm{A}}\right.$ of $1-$ acylpiperidine ring), 3.62-3.67 $\left(2 \mathrm{H}, \mathrm{m}, 2,6-\mathrm{CH}_{\mathrm{B}}\right.$ of 1-acylpiperidine ring), $5.21\left[1 \mathrm{H}, \mathrm{q}, J=7 \mathrm{~Hz},-\mathrm{NHC} \underline{\mathrm{H}}(\mathrm{CON}=) \mathrm{CH}_{2} \mathrm{~N}=\right], 7.20(1 \mathrm{H}$, br d, $J=7 \mathrm{~Hz}$, $\mathrm{NH}), 7.40-7.43(2 \mathrm{H}, \mathrm{m}, 3,5 \mathrm{H}$-aromatic $), 7.46-7.49(1 \mathrm{H}, \mathrm{m}, 4 \mathrm{H}$-aromatic), $7.80-7.82\left(2 \mathrm{H}, \mathrm{m}, 2,6 \mathrm{H}\right.$-aromatic). ${ }^{13} \mathrm{C}-\mathrm{NMR}\left(\mathrm{CDCl}_{3}\right) \delta: 24.2(\mathrm{C} 4$ of piperidine ring), 24.5 (C3 of 1-acylpiperidine ring), 25.6 (C4 of 1acylpiperidine ring), 26.1 (C3, C5 of piperidine ring), 26.6 (C5 of 1acylpiperidine ring), 43.4 (C2 of 1-acylpiperidine ring), 47.0 (C6 of 1acylpiperidine ring $), 47.6\left[-\mathrm{NH} \underline{\mathrm{CH}}(\mathrm{CON}=) \mathrm{CH}_{2}-\right], 55.1(\mathrm{C} 2, \mathrm{C} 6$ of piperidine ring), $61.8\left[-\mathrm{NHCH}(\mathrm{CON}=) \underline{\mathrm{CH}}_{2}-\right], 127.1(\mathrm{C} 2, \mathrm{C} 6$-aromatic $), 128.4$ (C3, C5-aromatic), 131.4 (C4-aromatic), 134.3 (C1-aromatic), 166.5 (ArCO), $170.1(=\mathrm{CH} \underline{\mathrm{CON}}=)$.

Acknowledgments We thank Dr. M. Fujiwara, Dr. K. Mishima, and Ms. Y. Iwase for their encouragement throughout this work.

\section{References and Notes}

1) For example, see Miyano S., Abe N., Takeda K., Fujisaki F., Sumoto K., Synthesis, 1982, 852 and references cited therein.

2) Prepn.: Miyano S., Abe N., Ger. Offen. 2323301 [Chem. Abstr., 80, $70539 h$ (1974)]; Pharmacology: Kase Y., Saita M., Takahama K., Masaki K., Miyata T., Jpn. J. Pharmacol., 24, 85-86 (1974).

3) Abe N., Fujisaki F., Sumoto K., Chem. Pharm. Bull., 46, 142-144 (1998).

4) Compound 7 is sparingly soluble in piperidine, and the reaction did not give the desired product under the same reaction conditions. Use of additional solvent resulted in the formation of unknown polymeric products.

5) Introductions of other amino functionalities instead of a piperidine ring substituent were also successful by the procedure via a mixed anhydride. These results together with pharmacological evaluation will be described elsewhere.

6) a) Bishoff S., Bittiger H., Delini-Stula A., Ortmann R., Eur. J. Pharmacol., 79, 225-232 (1982); b) Chivers J. K., Gommeron W., Leysen J. E., Jenner P., Marsden C. D., J. Pharm. Pharmacol., 40, 415-421 (1988); c) Jenner P., Elliott P. N. C., Clow A., Reavill C., Marsden C. D., ibid., 30, 46-48 (1978).

7) Dumuis A., Sebben M., Bockaert J., Naunyn-Schmideberg's Arch. Pharmacol., 340, 403-410 (1989).

8) The computational molecular model examinations were carried out by "overlay" operation combined with MM2 calculation. Some of the compounds in this series showed significant dopamine antagonist activities. These results will be described elsewhere. 